\title{
Les agréments, certifications, intercomparaisons
}

\author{
A. BIAU
}

Comme nous l'avons vu plus haut, le premier texte relatif aux conditions de mise en œuvre de la dosimétrie individuelle des travailleurs est l'arrêté du 19 avril 1968 pris en application du décret du 15 mars 1967. Les employeurs devaient déclarer le nom du laboratoire effectuant la surveillance de son personnel et étaient responsables de la qualité de ce suivi. Le SCPRI était susceptible de vérifier la qualité des mesures mais en pratique les contrôles effectifs et réguliers ne sont intervenus qu'après la publication du décret du 28 avril 1975 relatif à la surveillance des travailleurs dans les installations nucléaires de base. Dès l'année 1976, deux sortes d'intercomparaisons ont été réalisées avec les laboratoires du CEA, de COGEMA et d'EDF.

\subsection{Intercomparaison « physique »}

Des échantillons de dosimètres étaient adressés au SCPRI par chaque laboratoire des exploitants ci-dessus. Ces dosimètres étaient exposés par le SCPRI au laboratoire du Bureau international des poids et mesures de Sèvres, dirigé alors par le Professeur André Allisy, à des doses connues et à différentes énergies de photons. Les dosimètres étaient ensuite retournés aux laboratoires pour interprétation et les résultats transmis au SCPRI.

Ce type d'intercomparaison a toujours donné d'excellents résultats et n'a pas été reconduit chaque année.

\subsection{Intercomparaison « réglementaire »}

Ce type d'intercomparaison était effectué chaque année par l'intermédiaire des médecins du travail des centres CEA de Saclay, Fontenay aux Roses, Grenoble et Cadarache, des établissements COGEMA de La Hague et Marcoule et de chaque CNPE d'EDF. Les laboratoires de COGEMA, CEA et EDF adressaient au SCPRI 20 dosimètres pour chaque centre dont ils assuraient la dosimétrie. Ces dosimètres étaient conditionnés au SCPRI (OPRI) dans une pochette scellée à double compartiment avec d'un côté le dosimètre du laboratoire et dans l'autre un 
dosimètre du SCPRI (OPRI). Ces « doubles » dosimètres étaient ensuite transmis au médecin du travail de chaque centre qui en gardait un comme témoin et distribuait les 19 autres à des travailleurs exposés. Ceux-ci devaient porter ces doubles dosimètres au niveau de la poitrine à côté de leur dosimètre réglementaire durant un mois. À la fin du mois, ils étaient retournés au SCPRI (OPRI) qui, après quelques vérifications, prenait ses dosimètres et retournait aux laboratoires leurs dosimètres pour traitement. Ensuite les résultats du SCPRI (OPRI) et des laboratoires étaient confrontés.

Les résultats étaient globalement en bon accord à de rares exceptions près dans des cas très particuliers résolus après enquête.

La courbe « en trompette » de Bohm (ICRP, 1982) sur la figure 12.1 montre la répartition des écarts relevés au cours des intercomparaisons de 1999.

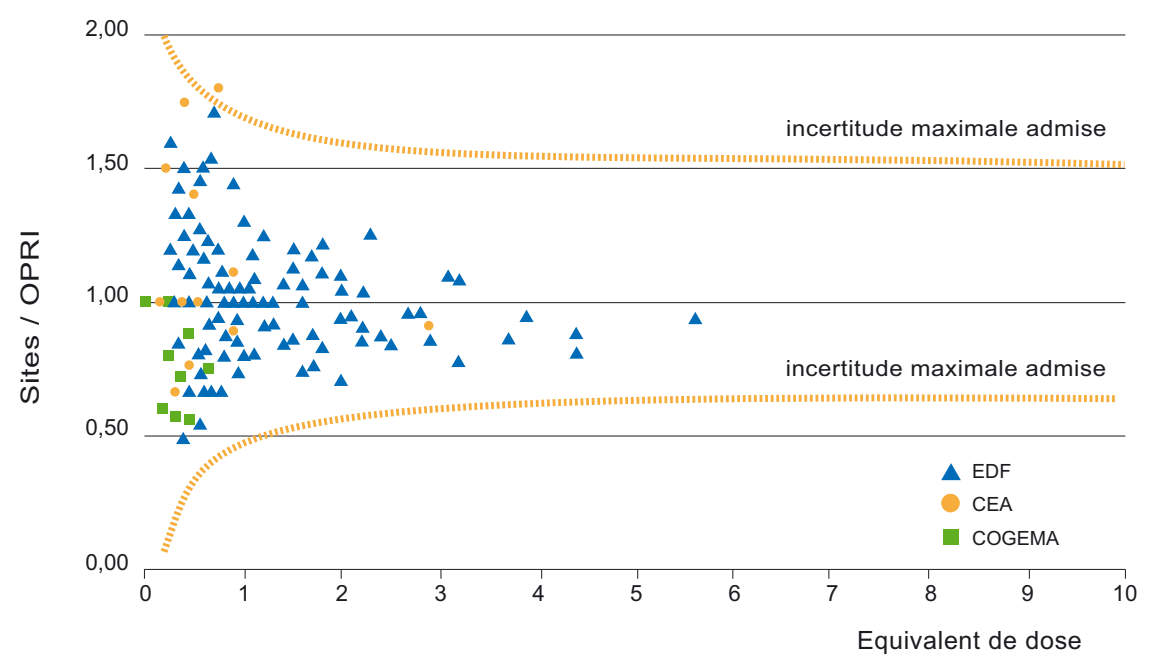

Figure 12.1 - Répartition des écarts relevés au cours des intercomparaisons de 1999.

En fonction des résultats de ces intercomparaisons, l'autorisation d'assurer la surveillance dosimétrique individuelle pour ses propres agents était accordée sous forme d'arrêté pris par le ministère du Travail après avis du Conseil supérieur de prévention des risques professionnels et du SCPRI (puis de l'OPRI).

Cette procédure s'est poursuivie jusqu'à la parution du décret du 31 mars 2003 et de l'arrêté du 6 décembre 2003 relatif à la délivrance du certificat et de l'agrément pour les organismes en charge de la surveillance des travailleurs qui sera modifié par arrêté du 21 décembre 2007. 
Toute l'organisation des contrôles des laboratoires en charge de la dosimétrie individuelle a commencé à se transformer à partir de l'arrêté de mars 1999 qui changeait le mode de suivi dosimétrique. En 2002, l'organisation de la radioprotection en France s'est elle-même transformée avec la création de la DGSNR (devenue ASN par la suite) et de l'IRSN. Enfin le décret du 31 mars 2003 et ses arrêtés d'application ont poursuivi cette transformation qui sera encore poursuivie quelques années plus tard.

\section{RÉFÉRENCE}

ICRP Publication 35 (1982) General Principles of Monitoring for Radiation Protection of Workers, Ann. ICRP 9, 4. 